\title{
INTERNET PACKET TRAFFIC CONGESTION
}

\author{
D.K. Arrowsmith ${ }^{\dagger}$, R.J. Mondrágon- $C^{*}$, J.M. Pitts * ${ }^{*}$ M.Woolf ${ }^{\dagger}$ \\ Mathematical Sciences ${ }^{\dagger}$, Dept of Electronic Engineering*, \\ Queen Mary, University of London, London E1 4NS, UK
}

\begin{abstract}
In recent years various models for packet traffic in networks have been developed. Most models have used Poisson-like sources which replicate traffic that is voice type. Such sources are said to be short-range-dependent $(S R D)$. The early models of Solé and Valverde demonstrated that longrange-dependent $(L R D)$ traffic could arise in the queue length dynamics at a given node within a communications network even when the sources are $L R D$ free. In this paper we consider the nature of the onset of congestion as load is increased in various types of network (regular, small-world and scale-free) and contrast their congestion behaviour by considering packet latency, or lifetime, indicators.
\end{abstract}

\section{INTRODUCTION}

We consider the behaviour of $L R D$ sources within packet traffic network models $[4,5,11]$ and show the erratic approach to congestion by comparison with $S R D$ traffic. The nature of $L R D$ is described and the traffic algorithm for the various networks is listed. The congestion properties of various types of regular lattice network are discussed. Scale-free networks and their congestion properties are also considered.

\subsection{Long-Range-Dependence}

$L R D$ was shown to be a feature of Internet packet traffic by Leland et al. in 1993, [7]. The LRD behaviour manifests itself along a communication channel as bursty activity in the packet rate (no of packets/unit time) which persists on all relevant time scales. The bursty traffic makes it much more difficult to implement effective traffic congestion protocols (TCP).

The statistical nature of $L R D$ traffic is formally defined in [3]. A key requirement is that the autocorrelation of binary packet traces, $c(k)$, where the lag is $k$, satisfies a power law decay of the form $c(k) \sim k^{-\beta}$, where $\beta \in(0,1)$. Equivalently, $c(k) \sim k^{-(2-2 H)}$, where $H=1-\beta / 2 \in(1 / 2,1)$ is the Hurst parameter, [3]. By comparison, $S R D$ traffic has an exponential rate of decay. The Hurst parameter distinguishes between $L R D$ traffic for $H \approx 1$ and the onset of short-range-dependent $(S R D)$ traffic for $H \approx 1 / 2$, when the autocorrelation decay changes to exponential.

The essential contrast between $S R D$, Poisson-like traffic, arising typically from traditional voice traffic, and the bursty nature of Internet $L R D$ traffic is seen in fig. 1 . The effect of scaling is shown for (a) short-range and (b) long-range dependent traffic for a time series of a random

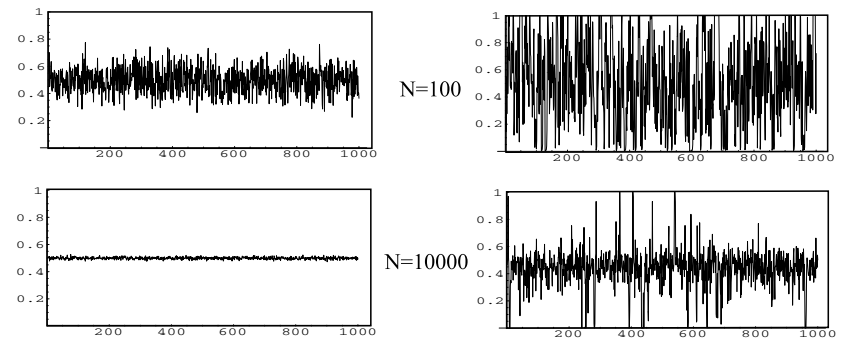

(a)

(b)

Figure 1. The batch averages of packets/unit time for (a) Poisson, and (b) $L R D$ sources, each for sizes $N=100$ and $N=10000$. A relatively large variance is retained in case (b).

variable $X_{n}, n=0,1,2, \ldots$ which takes binary values, i.e. $X_{n} \in\{0,1\}$. The data is averaged in batch sizes of $N=100$ and $N=10000$.

The standard deviation in the $S R D$ traffic varies as the square root of the batch size, or magnification, and we see a 'smoothing' of the traffic as $N$ increases in fig. 1(a). Thus the mean is an increasingly effective indicator of the instantaneous load, i.e expected packet rate, in the traffic. By comparison, for $L R D$ traffic, we see that the variation around the mean remains relatively high for large $N$ in fig. 1(b). Even when averaged over longer time intervals by several orders of magnitude, we still see extreme packet rates which are close to 0 and 1.

$L R D$ traffic increases queue lengths and delays dramatically, and they cannot necessarily be 'removed' by introducing $S R D$ data streams. The effects of $L R D$ need to be allowed for, both in computer models of network behaviour and in the routing algorithms used to control data flow through networks. Several lattice models with different topologies and routing algorithms have been studied for the emergence of congestion, $[4,5,11,13]$. In particular, Solé and Valverde [11], showed how the $L R D$ can arise from network interactions with $L R D$-free traffic hosts. In this paper, we compare Poisson sources with $L R D$ sources at the same load values, on various networks, which allows a closer study of the 'hierarchical' nature of $L R D$ from various sources, and models the real situation more closely. The implementation of control of host queue lengths also brings new insights into the model's behaviour, [13]. 


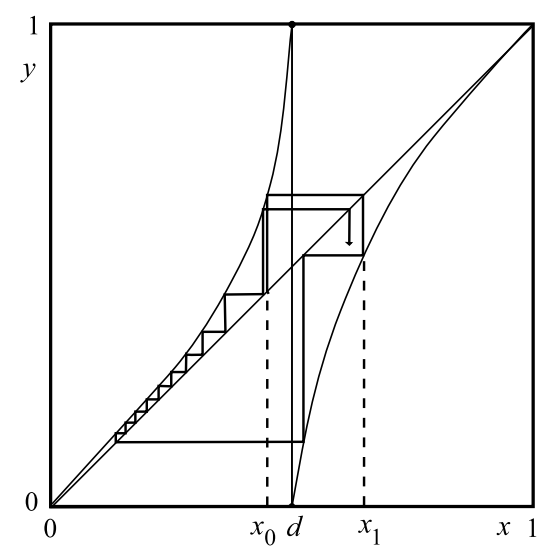

Figure 2. The graph of the map $f$ consists of two segments and each has a tangency with the line $y=x$. The iteration of the map $f$ with initial condition $x_{0}$ forms a 'web' generating the iterative sequence, or orbit, $x_{n}$, where $x_{n}=f\left(x_{n-1}\right), n=1,2, \ldots$ Note that the tangencies at $x=0,1$ gives a 'slow' change in the values of the sequence $x_{n}$, and therefore the output $z_{n}$ provides long sequences of consecutive '0's or ' 1 's.

\subsection{Nonlinear modelling of $L R D$}

A traffic map, [2], is used to provide binary sequences that model host packet production, see section 2 . The output ' 1 ' indicates that a packet is added to the host queue for transmission, otherwise ' 0 ' indicates that no packet is produced. The map $f$ is defined on the unit interval of real numbers $I=[0,1]$. The iteration of $f$ with an initial value $x_{0}$ produces an orbit $\left\{x_{n}\right\}$ defined by $x_{n+1}=f\left(x_{n}\right)$ in $I$, for $n=0,1,2, \ldots$ The sequence $\left\{x_{n}\right\}$ in $I$ is converted to binary output $z_{n}$ by associating the symbols ' 0 ' or ' 1 ' to the intervals $[0, d],(d, 1]$ respectively.

We use the family of Erramilli maps, [2], $f=f_{\left(m_{1}, m_{2}, d\right)}$ : $I \rightarrow I$, where

$$
f(x)= \begin{cases}x+(1-d)(x / d)^{m_{1}}, & x \in[0, d], \\ x-d((1-x) /(1-d))^{m_{2}}, & x \in(d, 1],\end{cases}
$$

see also related maps in $[10,12]$. Here $d \in(0,1)$ and the parameters $m_{1}, m_{2} \in[3 / 2,2]$ induce intermittency, of order $m_{1}$ and $m_{2}$, at the points $x=0$ and $x=1$ respectively. The intermittency produces slowly incremental streams of orbital values, [10]. This, in turn, ensures the so-called 'memory', or $L R D$, in the digital output $z_{n}$. It has been shown that the auto-correlation of the output function $z$, $c(k) \sim k^{-\beta}, k \in \mathbf{Z}^{+}$, has the decay constant $\beta=(2-$ $m) /(m-1) \in(0,1)$ with $m=\max \left\{m_{1}, m_{2}\right\},[6,8]$.

\section{COMMUNICATION NETWORKS}

The basic model we consider here, [11], has a lattice network of interconnected nodes which are either hosts or routers. All nodes can transfer packets and, additionally, hosts can both transmit and receive packets. Both types of node have buffers for storing packets. Packets when produced are allocated another host destination which is chosen randomly.
When a packet arrives at the head of a buffer, it then transfers to the queue at an adjacent node which is closer to its destination. Performance factors such as average delivery time of packets and the throughput of packets can now be considered for this model.

\subsection{Routing Algorithms}

A routing algorithm is needed to model the dynamic aspects of the network. Packets are created at hosts and sent through the lattice one step at a time until they reach their destination host. In real packet-switching networks, packets carry header and information payloads with them, including data about the state of the network. To simplify the modelling, we record only the time of creation and the source and destination addresses when passing packets through the network.

The routing algorithm operates as follows:

- first a host creates a packet using either a uniform random distribution (Poisson) or a distribution defined by a chaotic map $(L R D)$, as described in section 1.

- if a packet is generated it is put on the end of the queue for that host. This is repeated for every host in the lattice.

- packets at the head of each queue are sent to a neighbouring node which is closer to the destination node (using the least used link if necessary).

This process is repeated for each node in the lattice. The whole procedure of packet generation and movement represents one time step of the simulation. Initially, there is no feedback implemented on queue lengths in this algorithm and hence the model is uncontrolled.

\subsection{Network Models}

The triangular and honeycomb lattice networks have now been investigated to check the robustness of the results for the rectangular grid in [13]. In each case, the density of hosts $\rho \in[0,1]$ is the ratio between the number of hosts and the total number of nodes in the network. Hosts are randomly distributed throughout the network and we fix $\rho=0.164$ for the simulations presented here. Poisson-like traffic is created by randomly choosing a number on the interval $[0,1]$ and if it is below a discriminator value $\lambda$, then a packet is emitted. Hence, for a uniform distribution the average rate at which packets are produced at a host is $\lambda$.

Rectangular lattice models of this type have been widely discussed, see $[5,9,11,13]$. The finite rectangular(R) lattice $\mathcal{Z}$ consists of $L^{2}$ nodes and each node has four neighbours. By comparison, Internet networks typically have the characteristics of both clustering and short path-lengths. Smallworld $(S W)$ networks introduced by Newman and Watts have these features. For example, the regular graph in fig. 3 (c) consists of an underlying double ring network with $S$ nodes and valency $m=4$, which is changed into a SWnetwork by the addition of random links with a probability $p$. For this network, the average path-length $d_{a v}(p)$ satisfies $d_{a v}(0) \approx \alpha \ln (S-\beta)+\delta$ and $d_{a v}(1)=\ln (S) / \ln (m)$.

Scale-free $(S F)$ networks, [1] and fig. 3(d), have the property that the probability, $P(k)$, of a given node having $k$ links has a power law decay, i.e. $P(k) \sim k^{-\gamma}$, with $\gamma \in(2,3)$ for the Internet. Depleted rectangular lattices, or 


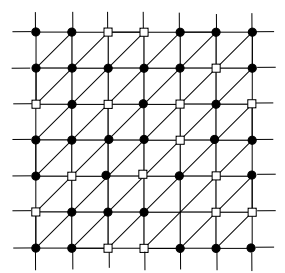

(a)

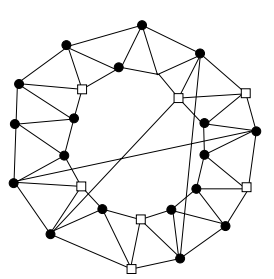

(c)

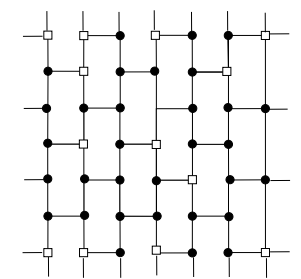

(b)

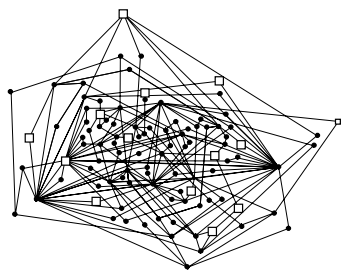

(d)
Figure 3. (a) Triangular(T), and (b) hexagonal $(\mathrm{H})$ toroidal lattices, used for testing congestion properties as super- and sub- sets of the R-lattice. (c) A regular ring network with valency $m=4$ is shown with added random connections. (d) The scale-free graph has been constructed with $\gamma=3$. Hosts $(\square)$ are distributed randomly in the network.

percolation graphs, where edges are removed randomly with a given probability, have also been considered - they form a useful collection of intermediate structures between the regular, fig 3.(a,b) and non-regular networks, fig. 3(c,d).

\section{CRITICAL LOAD BEHAVIOUR}

A key factor in the network which has a pronounced effect on congestion is the packet rate of production at the hosts. The obvious control is to reduce the rate. This can have a dramatic effect for $S R D$ traffic but is not so useful in the $L R D$ case. Performance indicators, such as packet lifetime, can change so dramatically as load is increased that they can sometimes be best described by a phase transition, $[1,4,9]$. If a critical load exists at which the delivery time of packets increases dramatically, then a clear control strategy to keep the load below criticality can be implemented. However, when the transition to congestion is less clear-cut, as we show for $L R D$ traffic, an effective control strategy becomes much more problematical.

Fig. 4(a) gives a comparison of onset of congestion in two otherwise identical networks with host density $\rho=$ 0.164 . The average lifetime, or end-to-end delay of a packet, is plotted against the load $\lambda$, the average number of packets generated per host per unit time. There is a phase transition from a free phase in which lifetimes remain small to a congested phase in which lifetimes increase rapidly. The hosts in one case are Poisson, and for the other cases, the hosts are $L R D$ with different values of the Hurst parameter. For simplicity, the intermittency parameters $m_{1}, m_{2}$ are kept equal (to $m$ ). When the exponents differ, the strongest intermittency dominates the auto-correlation behaviour, $[6,8]$. The nature of the traffic changes from $S R D$ at $m=1.5(H=0.5)$ to full $L R D$ at $m=2(H=1)$. The most pronounced differences do occur near and immediately below the critical load point, $\lambda=\lambda_{c}$. Crucially, for $\lambda<\lambda_{c}$, the average lifetimes for $L R D$ sources are much greater than for Poisson sources - typically by orders of magnitude, [13]. The comparison of onset of criticality for other types of network is seen if fig. 5 .
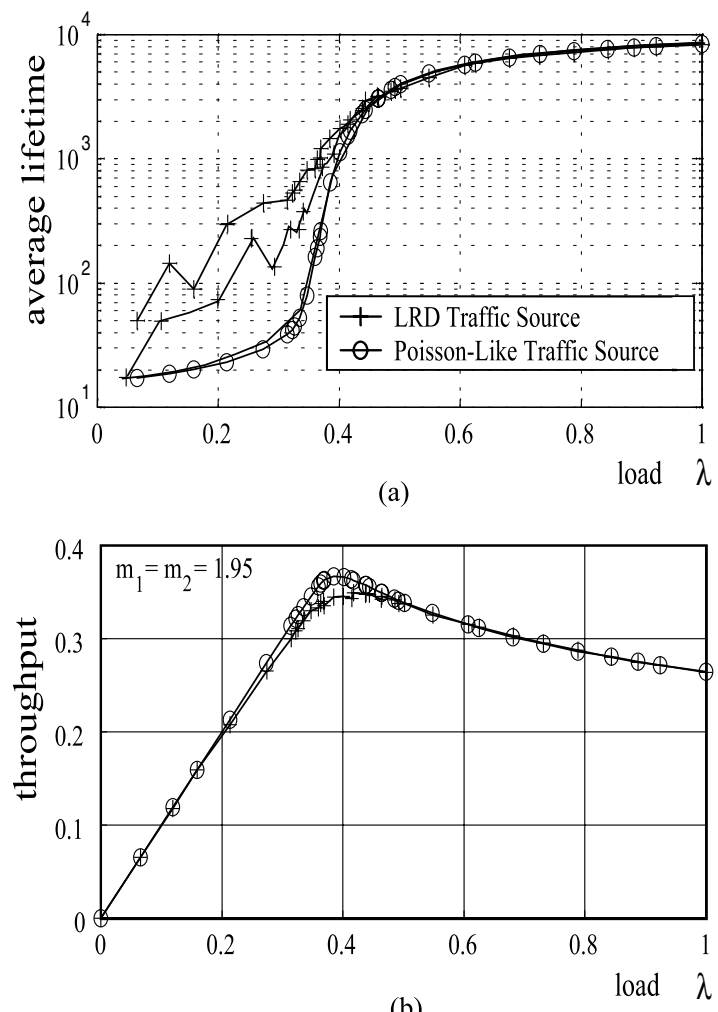

(b)

Figure 4. (a) A smooth transition to congestion is shown for the Poisson sources. As $L R D$ is introduced, by taking $m>1.5$ in eqn.(1) we see an increasingly rapid rise in the average lifetime of packets for lower loads. (b) The throughput increases up to the Poisson critical load of $\lambda=$ 0.39 , but the maximum throughput achieved is lower for $L R D$ traffic.

In Fig. 4(b) the peak in throughput occurs at the critical point where the network reaches its peak efficiency. The peak value of throughput, is slightly lower for the $L R D$ sources, emphasizing the longer lifetimes of packets. However, the difference is less pronounced than that seen in average lifetimes. Away from the peak, values of throughput for the two types of traffic source are very similar. Although the throughput is only slightly reduced, the average lifetimes increase by up to a factor of ten. This earlier congestion onset appears to be the most important feature of the model, and has significant implications for shared backbone data network infrastructures.

\subsection{Mean Field Models}

It should be noted that as $\rho$ increases, the total load on the system increases and the phase transition becomes sharper. 


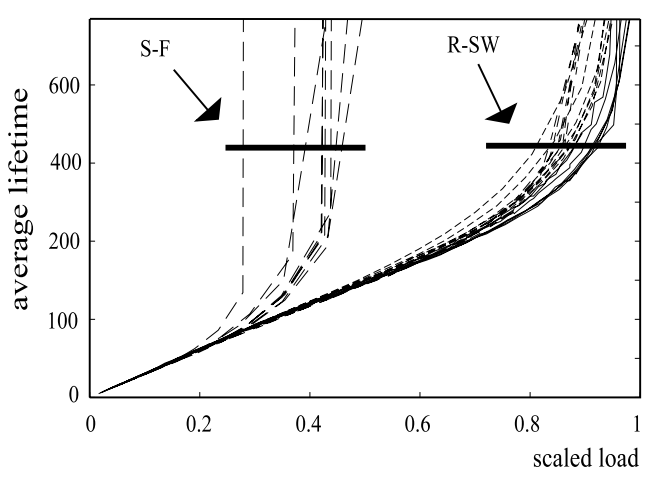

Figure 5. The critical transitions for average packet lifetime in SF networks occur at substantially lower loads than those for both regular and SW networks.

In fact, simple mean field approaches [13] suggest the reciprocity property $\lambda_{c}=1 / \rho d_{a v},[5,11,13]$, for the critical load, which can be obtained by considering total distance' to destination' properties, see fig. 6. This result was obtained for the R-lattice (for the special case $\rho=1$ ) in [5]. The load at which congestion begins, $\lambda_{c}^{\prime}<\lambda_{c}$ requires more sophisticated estimates and is obtained by distinguishing the host and router traffic, [13].

There is strong evidence that the mean field approach becomes an increasingly poor predictor of criticality at low loads for depleted regular graphs.

\subsection{Packet Traffic Simulation with Control}

The simplest way to control packet traffic is to limit the length of queues. Long queues in the network invariably occur at hosts and a control mechanism was considered to reduce the rate of packet production at hosts with long queues. The simulation keeps count of packets produced, so the actual or 'carried' load is known. Application of this simple control mechanism, [13], showed that the network can be prevented from becoming congested by effectively limiting its packet carrying capacity. This is particularly useful in the $L R D$ case when congestion can occur at much lower load levels. Extra refinements in routing and network information carried by the packet are being considered, see also [4] for recent progress.

Current work aims to develop the uncontrolled model into a controllable model which reacts to the local build-up of queues with an objective of delaying onset of congestion at the network level when the sources are strongly longrange dependent.

\section{REFERENCES}

[1] R. Albert and A-L Barábasi, Statistical Mechanics of Complex Networks, Rev. Mod. Phys. 74, 47 (2002), also arXiv:cond-mat/0106096, 1-54.

[2] A. Erramilli, R.P. Singh and P. Pruthi, Chaotic maps as models of packet traffic, Proc. $14^{\text {th }}$ Int. Teletraffic Conf. 1994, North-Holland(Elsevier), 329-38.

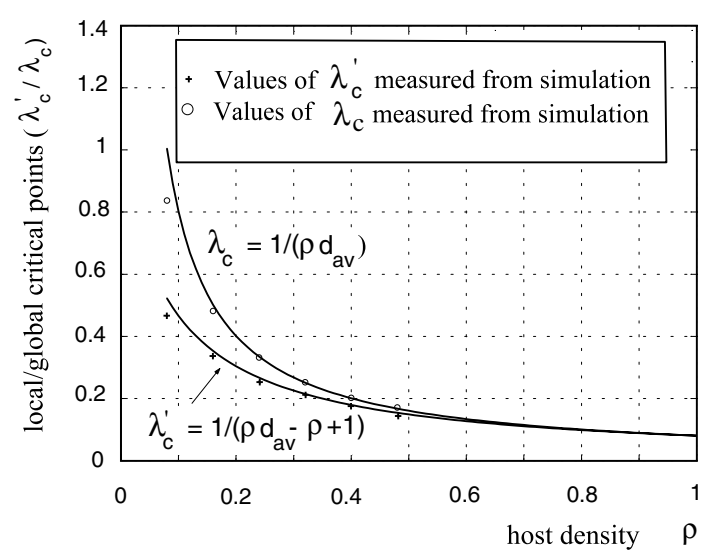

Figure 6. Mean field approximations using a total packet "distance function" for critical load $\lambda_{c}$, and the onset of critical load $\lambda_{c}^{\prime}=1 /\left(\rho d_{a v}-\rho+1\right)$ showing strong agreement with numerical simulations for the triangular lattice. Similar behaviour occurs for the R- and H- lattices with increasingly low critical loads as the lattice becomes sparser.

[3] J. Beran, Statistics of long memory processes, Monographs on Stats and Appl. Prob. 61, Chapman \& Hall, London.

[4] K.Fukuda, H.Takayasu, and M.Takayasu, Origin of Critical Behavior in Ethernet Traffic, Physica A 287, 2000, 289-301.

[5] H. Fǔks and A.T. Lawniczak, Performance of data networks with random links, Math and Computers in Simulation, 51 1999, 101-17.

[6] A. Giovanardi, G. Mazzini, and R. Rovatti, Chaos based self-similar traffic generators, Proc. NOLTA 2000, 747-50.

[7] W.E. Leland, M.S. Taqqu, W. Willinger and D.V. Wilson, On the Self-Similar Nature of Ethernet Traffic, Proc ACM SIGCOMM 93, 1993.

[8] R.J. Mondragón, A model of packet traffic using a random wall model, Int. Jnl of Bif. and Chaos, 9(7), 138192, 1999.

[9] T. Ohira and R. Sawatari, Phase transition in a computer network traffic model, Phys. Rev. E 58 1998, 193-95.

[10] H.G. Schuster, Deterministic Chaos: An Introduction, $3^{\text {rd }}$ Edition VCH, 1995.

[11] R. V. Solé and S. Valverde, Information transfer and phase transitions in a model of internet traffic, Physica A 289, 2001, 595-605.

[12] X-J Wang, Statistical physics of temporal intermittency, Phys Rev A 40(11), 1989, 6647-61.

[13] M. Woolf, D.K. Arrowsmith, R.J. Mondragon, J.M. Pitts, Optimization and Phase Transitions in a Chaotic Model of Data Traffic, Phys Rev E 66, 046106 (2002). 